\title{
Les Difficultes Inherentes A La Mobilisation Des Connaissances Mathematiques Dans La Physique, Cas De La Mecanique Au College
}

\begin{abstract}
Khadija Raouf
Enseignant chercheur et formatrice des futurs enseignants des sciences physiques

Observatoire de recherche en didactique et pédagogie universitaire Centre Régional des Métiers de l'Education et de la Formation d'Eljadida, Maroc
\end{abstract}

Imad Belazzaar Morad Radi

Enseignants des sciences physiques, cycle de l'enseignement secondaire collégial Ministère de l'Education Nationale et de la Formation

Professionnelle, Maroc

\section{Mohamed Moussetad Mohammed Talbi}

Enseignants chercheurs, Observatoire de recherche en didactique et pédagogie universitaire Faculté des sciences Ben M'Sik. Hassan II

University, Casablanca, Maroc

doi: 10.19044/esj.2016.v12n25p185 URL:http://dx.doi.org/10.19044/esj.2016.v12n25p185

\begin{abstract}
This study focuses on the difficulties of pupils in final year of college cycle (14-17 years) to mobilize mathematical knowledge in mechanical modeling activities. The objective is to try to understand the dynamics of the transfer of mathematical knowledge in modeling the concept of "speed" and the notion of "force." To do this, we developed two individual written questionnaires to a sample of pupils. One focuses on mathematics and the other mechanics. To better interpret some results, we held individual interviews with a sample of pupils. Analysis and interpretation of the results of the questionnaires and interviews show that the mathematical knowledge mobilization difficulties are due in the first hand to the non mastery of this knowledge in mathematic courses and the second hand to the fact that pupils aren't conscious of the linkages which exist between mathematic and physic. The students having answered correctly seem adopting memorization strategies than cognitive strategies conducive to the transfer.
\end{abstract}


Keywords: Transfer, modelization, mobilization, transdisciplinary

\section{Resume}

Cette présente étude porte sur les difficultés des élèves de la dernière année du cycle collégial (14-17 ans) à mobiliser des connaissances mathématiques dans des activités de modélisation mécanique. L'objectif étant d'essayer de comprendre la dynamique du transfert de ces connaissances mathématiques dans la modélisation de la notion «vitesse » et de la notion «force ». Pour ce faire, nous avons élaboré deux questionnaires individuels écrits destinés à un échantillon d'élèves. L'un porte sur les mathématiques et l'autre sur la mécanique. Pour mieux interpréter certains résultats, nous avons organisé des entrevues individuelles avec un échantillon d'élèves. L'analyse et l'interprétation des résultats des deux questionnaires écrits ainsi que ceux des entrevues montrent que les difficultés de mobilisation des connaissances mathématiques sont dues d'une part à la non maitrise de ces connaissances dans la situation initiale d'acquisition et d'autre part à la quasi absence de reflexes d'articulation et de liens entre la situation source et la situation cible. Les élèves qui répondent correctement semblent adopter des stratégies de mémorisation au dépend de stratégies cognitives propices au transfert.

Mots-clés : Transfert, modélisation, mobilisation, transdisciplinarité

\section{Introduction}

Le système éducatif marocain a connu depuis l'indépendance une dynamique de mutations profondes ayant permis de réaliser des progrès notables au niveau de la politique de l'éducation, de l'architecture de l'enseignement, des approches pédagogiques, des curricula ainsi qu'au niveau de la formation initiale et continue des différents intervenants (Chafiqui et Alagui, 2011). Malgré ces efforts, des dysfonctionnements chroniques limitent leurs effets qualitatifs au niveau de l'acquis des apprenants et des pratiques pédagogiques (TIMSS, 2011 ; CSEFRS, 2009 et 2014 ; MENFP, 2014 ; Unesco, 2014).

Pour capitaliser les acquis et dépasser ces dysfonctionnements qui affectent négativement les missions de l'école, le Maroc s'est doté d'une vision stratégique qui s'étend sur la période 2015-2030. Parmi les changements majeurs visés sur le plan pédagogique, on note le passage de la logique de la transmission des savoirs et de la mémorisation à une logique de l'apprentissage. Comme souligné par Lauzon (2000), l'apprentissage ne se limite pas à l'acquisition de connaissances et à leur application mais suppose aussi la capacité de les réinvestir dans la résolution des problèmes, dans la réalisation des projets ou dans l'accomplissement de tâches d'ordre cognitif, 
socio-affectif ou psychomoteur. Pour ce faire, le sujet devrait être en mesure d'utiliser de manière pertinente l'outil cognitif en l'associant à des situations dans lesquelles il aura identifié un problème pour lequel il connaît son efficacité (Meirieu, 1991) ce qui favorise des apprentissages signifiants et intégrés.

Dans cette perspective, il est essentiel de situer au cœur de l'acte enseignement-apprentissage la notion du transfert qui constitue, à coté de l'acquisition et de la rétention, les trois paramètres principaux par lesquels on évalue la qualité d'un apprentissage (Péladeau et al, 2005). A juste titre, Bracke (1998) écrit que le but ultime de l'enseignement est d'amener les élèves à transférer leurs apprentissages d'une tâche à une autre, d'une année scolaire à une autre, de l'école à la maison et du milieu scolaire à celui du travail.

Dans cet article, nous nous sommes intéressés à la dynamique de mobilisation des connaissances mathématiques dans des situations de modélisation mécanique en physique par des collégiens marocains. En premier lieu, nous présentons le cadre conceptuel de notre étude basé sur une recherche bibliographique sur la question du transfert des connaissances puis la problématique et le contexte de la recherche. En troisième lieu, nous présentons la méthodologie adoptée et les outils d'investigations utilisés suivi d'une section qui porte sur les résultats de l'étude et de leur analyse. Nous concluons en mettant en exergue les principales difficultés relatives à la mobilisation des connaissances mathématiques en mécanique ainsi que les facteurs pouvant les favoriser.

\section{Cadre Conceptuel}

La question du transfert des apprentissages préoccupent plusieurs chercheurs anglo-saxons et francophones depuis plus d'une décennie (Bracke, 1998 ; Brouillette, 2002 ; Forcier et Goulet, 1996; Haskell, 2001 ; Gagné, 1962 ; Gick et Holyak, 1987; Mendelson et Anderson, 1990 ; Mc.keough et al, 1995 ; Moffet ,1995 ; Perrenoud, 1997, 2000 ; Presseau et Frenay, 2004, Tardif,1992, 1999; Tardif et Meirieu, 1996, ; Singley,1989; Thorndike, 1906; ).

Le transfert est en général défini comme étant la capacité d'un sujet à réutiliser d'une façon judicieuse et fonctionnelle des connaissances ou des compétences construites lors d'une situation initiale (source) dans une nouvelle situation (cible) (Brake, 1998; Perrenoud, 1997 ; Tardif et Meirieu, 1996; Tardif et Presseau, 1998; Tardif, 1999).

Comme dans le transfert, il ne s'agit pas de se référer à n'importe quelles connaissances mais plutôt à une structure de connaissances de haut niveau, il est nécessaire que le sujet reconnaisse d'abord, les ressemblances qui existent entre la situation source et la situation cible. Or comme le 
soulignent Tardif et Presseau (1998) bien que des situations présentent de nombreuses similitudes, souvent, elles ne sont pas perçues par les sujets et par conséquent le transfert n'est pas effectué. Ces constats confirment ceux de Gick et Holyak $(1980 ; 1983)$ qui ont montré que le transfert se fait rarement d'une façon spontané. Par contre si les sujets sont informés du lien qui existe entre les deux situations, la probabilité du transfert augmente sensiblement.

La mise en correspondance des éléments de la situation cible et de la situation source est procédée selon le modèle théorique de tardif (Tardif, 1999) relatif à la dynamique du transfert par 3 sous processus. Le premier concerne l'encodage des apprentissages dans la situation source oŭ le sujet réalise que les connaissances construites ou les compétences développées seront éventuellement réutilisées dans d'autres contextes. Le deuxième sous processus est celui de la représentation que le sujet construit à propos de la situation cible: les buts escomptés, les éléments à prendre en compte dans la résolution de la dite situation, les contraintes. Le troisième sous processus est celui de l'accessibilité aux connaissances ou compétences en mémoire à long terme à mobiliser pour résoudre la situation cible.

Ces trois sous processus sont tributaires du niveau de maitrise des connaissances à transférer et de la représentation que le sujet en construit, d'où l'importance accordée à la façon dont le savoir a été construit dans le contexte initial d'acquisition (Tardif et Presseau, 1998). Ces mêmes auteurs considèrent que le degré de similitude entre la tâche source et la tâche cible, la capacité du sujet à reconnaître ces similitudes existant entre le contexte initial d'acquisition et le contexte cible et enfin, le contexte d'apprentissage de la situation source sont les principaux facteurs influençant la qualité du transfert.

\section{Problematique Et Contexte De La Recherche :}

Les travaux de cet article se situent dans la continuité d'une recherche, dont les résultats sont soumis pour publication (Raouf et al), que nous avons menée auprès d'un échantillon de professeurs des sciences physiques et professeurs des mathématiques exerçant 20 ans et plus dans l'enseignement secondaire collégial au Maroc. L'étude s'est basée sur un questionnaire individuel qui a permis de dégager:

1. le point de vue des enseignants sur les difficultés rencontrées par les collégiens marocains en dernière année du cycle, dans l'apprentissage, des premières notions de mécanique et des notions mathématiques mobilisées dans la modélisation des concepts « vitesse » et « force ».

2. Certains aspects des pratiques enseignantes déclarées relatives à l'enseignement des connaissances mathématiques mobilisées dans les activités de modélisation en mécanique, à la dynamique d'exploitation de ces connaissances dans le cours de la physique et à la qualité des rapports de 
collaboration et de cogestion de ces connaissances par les professeurs des deux disciplines.

Selon les déclarations de la majorité des professeurs des sciences physiques interrogés, les élèves ont des difficultés à mobiliser leurs connaissances mathématiques dans le contexte de la mécanique à cause de leur faible niveau en mathématique, ce qui ne facilite pas la tâche aux professeurs surtout que les élèves étudient les notions de mécanique pour la première fois.

Les enseignants de mathématiques confirment ce constat qu'ils justifient par la non-maitrise des pré-requis abordés au primaire ce qui entrave le développement de nouveaux apprentissages au collège.

Ces déclarations des enseignants ne semblent pas être une spécificité marocaine, d'ailleurs Tardif et Meirieu (1996) soulignent qu'il est fréquent d'entendre des enseignants des différents cycles (primaire, secondaire, universitaire) et de différentes disciplines se plaindre d'une part, de la faible rétention par les élèves des connaissances abordées dans le cycle antérieur et d'autre part de leurs difficultés à réutiliser leurs connaissances construites dans une autre discipline. L'analyse et l'interprétation des déclarations des professeurs des mathématiques et des sciences physiques relatives à leurs pratiques enseignantes dans la gestion des notions transversales ont révélé que les pratiques ainsi que le contexte de travail (organisation pédagogique, le volume imparti, etc.) semblent favoriser la territorialisation des disciplines et par conséquent, handicaper la mise en œuvre du processus de transfert ou des démarches transdisciplinaires.

A ces résultats, s'ajoutent les constats relevés lors de l'accompagnement des futurs professeurs dans leurs stages professionnels effectués dans les établissements scolaires qui montrent des pratiques pédagogiques favorisants la fragmentation des apprentissages et misant sur l'enseignement de contenu au dépend de développement chez l'élève des stratégies d'apprentissage. Ce genre de pratiques est en grande partie responsable de la rareté des transferts (Perrenoud, 1997; Tardif, 1999) ainsi que la territorialisation des disciplines (Haskell, 2001).

Partant de ce contexte, nous nous sommes donc intéressés à l'examen de la dynamique de transfert de certaines notions mathématiques (calcul algébrique et la géométrie vectorielle) dans des situations simples de modélisation mécanique (la vitesse et les actions mécaniques).

Nous entendions par cette étude, dégager des éléments pouvant éventuellement nous éclairer sur les difficultés des élèves à mobiliser des connaissances acquises dans une situation source (en cours de mathématique) dans une situation cible (en mécanique) et sur les facteurs handicapant ce transfert en essayant de répondre aux questions suivantes :

- Les élèves maitrisent-ils les connaissances à mobiliser d'abord en situation source? 
- Les élèves arrivent-ils à dégager les liens existants entre la situation source et la situation cible ?

- $\quad$ Les élèves se basent-ils sur des stratégies de mémorisation ou sur des stratégies axées sur la transférabilité ?

Nous avons fait le choix de travailler avec des élèves de la dernière année du cycle du secondaire collégial pour les considérations suivantes :

- Le cycle du secondaire collégial constitue d'une part, la transition entre le cycle primaire et le cycle du secondaire qualifiant (lycée). Le collège est un lieu de consolidation des connaissances abordées au primaire et la construction de nouveaux apprentissages qui auront leurs prolongements au lycée.

- C'est une étape déterminante dans le choix de l'orientation des élèves.

Notre objectif n'est pas de limiter la notion du "Transfert" à un raisonnement analogique ou de réduire les problématiques liées à l'apprentissage de la physique à un problème de mathématique, mais nous cherchons à comprendre le mécanisme cognitif des sujets à traiter des situations de transfert simples présentant des structures logiques homologues.

\section{Elements methodologiques}

L'étude, objet de cette publication, s'intéresse aux difficultés des collégiens marocains à traiter des relations algébriques liant des grandeurs physiques en mécanique et à réaliser des activités de modélisation simples liées au concept " force". Toutes ces activités nécessitent la mobilisation de connaissances abordées en mathématique (calcul algébrique et géométrie vectorielle). Pour ce faire, nous avons de prime abord procéder à l'analyse de la structure et du contenu du programme de mécanique à partir des instructions pédagogiques officielles relatives au programme des sciences physiques au collège (version mars 2015) puis nous nous sommes intéressés aux notions mathématiques engagées dans les activités de modélisation que nous avons déterminées à partir de l'analyse de quatre manuels scolaires (Almofid dans les sciences physiques, Almohit dans les sciences physiques, ouahat des sciences physiques, Almounir dans les sciences physiques).

Ensuite, nous avons élaboré un questionnaire écrit de physique puis un questionnaire écrit en mathématique que nous avons soumis à 66 élèves de la $3^{\text {ème }}$ année du secondaire collégial de l'année 2014-2015. Les items ont été construits à partir des résultats d'analyse des documents officiels et des résultats soumis pour publication mentionnée dans la section précédente (Raouf et al). Pour mieux interpréter les résultats et afin de dégager des éléments de réponses à la 3éme question de la recherche, nous avons organisé des entrevues individuelles semi dirigées avec une vingtaine de sujets et avec qui, nous avons discuté les principaux résultats de l'étude. 


\section{Les questionnaires écrits}

Les deux questionnaires sont conçus de façon à ce que chaque item de la situation source (mathématiques) a son homologue en situation cible (physique). Cette similarité se manifeste au niveau de la présentation et du type de traitement des situations sources et des situations cibles.

Le questionnaire de mathématique porte sur les connaissances mathématiques mobilisées dans les situations de modélisation en cours de mécanique et qui ont été cité par les professeurs de mathématiques et des sciences physiques interrogés (Raouf et al) comme présentant des difficultés d'apprentissage pour les collégiens de $3^{\text {ème }}$ année. L'objectif étant de vérifier l'étendue de leur maitrise dans la situation source (Toussaint, 1996) et par conséquent d'essayer de répondre à la $1^{\text {ère }}$ question de la recherche.

Le questionnaire de physique concerne des situations de mécanique mobilisant d'une part, le traitement algébrique des relations qui concernent les notions "vitesse", "distance" et " durée" et d'autre part, l'exploitation de la géométrie vectorielle pour représenter la force $\mathrm{F}$ et expliciter la condition d'équilibre d'un corps soumis à deux forces. Le choix des items a été motivé par les résultats du dépouillement du questionnaire adressé aux professeurs des sciences physiques qui attestent que les élèves ont des difficultés à mobiliser leur pré-requis mathématiques (calcul algébrique et géométrie vectorielle) dans des situations simples de mécanique (Raouf et al). L'objectif étant d'essayer de dégager les difficultés des élèves, sujets de l'expérimentation, à mobiliser leurs connaissances mathématiques dans le traitement des situations mécaniques liées à la notion de la vitesse abordée dans la première unité du cours de mécanique (mouvement et repos) et de la notion "force" abordée dans la deuxième et la troisième unités du cours de mécanique, respectivement, les actions mécaniques et le concept force. Les situations auxquelles les élèves ont été confrontés sont identiques à celles déjà abordées dans le programme de chaque discipline, il en est de même pour la formulation des consignes et les apprentissages à mobiliser sont strictement scolaires. Les items constituants les deux questionnaires sont consignés dans le tableau 1 ci-dessous :

\begin{tabular}{|c|c|c|c|}
\hline $\begin{array}{c}\mathrm{N}^{\circ} \text { de } \\
\text { l'item }\end{array}$ & $\begin{array}{c}\text { Items de } \\
\text { mathématique }\end{array}$ & Items de physique & $\begin{array}{c}\text { Notions mathématiques à } \\
\text { mobiliser }\end{array}$ \\
\hline & $\begin{array}{c}\text { Exprimez b en } \\
\text { fonction de a et c à } \\
\text { partir de la relation } \\
\mathrm{a}=\mathrm{b}+\mathrm{c}\end{array}$ & $\begin{array}{c}\text { Exprimez la distance de } \\
\text { réaction }\left(\mathrm{D}_{\mathrm{R}} \text { en fonction de }\right. \\
\text { la distance de freinage }\left(\mathrm{D}_{\mathrm{F}}\right)\end{array}$ & $\begin{array}{c}\text { Calcul algébrique } \\
\text { et de la distance d'arrêt } \\
\left(\mathrm{D}_{\mathrm{A}}\right) \text { à partir de la relation } \\
\text { (addition/multiplication) }\end{array}$ \\
& & $\mathrm{D}_{\mathrm{A}}=\mathrm{D}_{\mathrm{R}}+\mathrm{D}_{\mathrm{F}}$ & \\
\hline
\end{tabular}




\begin{tabular}{|l|c|c|c|}
\hline 2 & $\begin{array}{c}\text { Exprimez c en } \\
\text { fonction de } \mathrm{a} \text { et } \mathrm{b} \text { à } \\
\text { partir de la relation } \\
\mathrm{a}=\mathrm{b} / \mathrm{c}\end{array}$ & $\begin{array}{c}\text { Exprimez temps } \mathrm{t} \text { à partir de } \\
\text { la relation } \mathrm{V}_{\mathrm{m}}=\mathrm{d} / \mathrm{t}, \text { où } \mathrm{V}_{\mathrm{m}} \text { et } \\
\text { d désignent respectivement } \\
\text { la vitesse moyenne et la } \\
\text { distance parcourue. }\end{array}$ & $\begin{array}{c}\text { Calcul algébrique } \\
\text { (multiplication/division) }\end{array}$ \\
\hline
\end{tabular}

\begin{tabular}{|c|c|c|c|}
\hline $\begin{array}{c}\mathrm{N}^{\circ} \text { de } \\
\text { l'item }\end{array}$ & $\begin{array}{c}\text { Items de } \\
\text { mathématique }\end{array}$ & Items de physique & $\begin{array}{c}\text { Notions mathématiques à } \\
\text { mobiliser }\end{array}$ \\
\hline 3 & $\begin{array}{c}\text { Exprimez X à partir } \\
\text { de } 9 \mathrm{a}=\mathrm{X}^{2}\end{array}$ & $\begin{array}{c}\text { Exprimez la vitesse de } \\
\text { freinage en fonction de } \\
\text { la vitesse à partir de la } \\
\text { relation } \mathrm{D}_{\mathrm{F}}=2 \mathrm{~V}^{2}\end{array}$ & $\begin{array}{c}\text { Calcul algébrique (racine } \\
\text { carrée) }\end{array}$ \\
\hline 4 & $\begin{array}{c}\text { Représentation d'un } \\
\text { vecteur à partir de } \\
\text { ses coordonnées }\end{array}$ & $\begin{array}{c}\text { Représentez la force à } \\
\text { partir de ces } \\
\text { caractéristiques }\end{array}$ & $\begin{array}{c}\text { Représentation d'un } \\
\text { vecteur à partir de ses } \\
\text { coordonnées }\end{array}$ \\
\hline 5 & $\begin{array}{c}\text { Expression de la } \\
\text { somme de deux } \\
\text { vecteurs opposés }\end{array}$ & $\begin{array}{c}\text { Exploitation des } \\
\text { conditions d'équilibre } \\
\text { d'un corps soumis à } \\
\text { deux forces }\end{array}$ & $\begin{array}{c}\text { Somme de deux vecteurs } \\
\text { opposés }\end{array}$ \\
\hline
\end{tabular}

Tableau 1

\section{Resultats et discussion :}

\section{Les notions mathématiques mobilisées dans la mécanique au collège :}

Le tableau ci-dessous résume les apprentissages visés par chaque unité du programme de la mécanique ainsi que les notions mathématiques mobilisées dans les activités de modélisation :

\begin{tabular}{|c|c|c|}
\hline Axe & Apprentissages visés & Notions mathématiques mobilisées \\
\hline $\begin{array}{c}\text { Mouvement } \\
\text { et repos }\end{array}$ & $\begin{array}{l}\text { Définition du référentiel } \\
\text { Description du mouvement } \\
\text { à partir de la forme de sa } \\
\text { trajectoire et de l'évolution } \\
\text { de la valeur de sa vitesse } \\
\text { Distinction entre le type de } \\
\text { trajectoire (rectiligne, } \\
\text { circulaire, curviligne) et le } \\
\text { type du mouvement } \\
\text { (translation, rotation). } \\
\text { Expression et calcul de la } \\
\text { vitesse moyenne } \\
\text { Les différentes natures du } \\
\text { mouvement (Mouvement } \\
\text { uniforme, décéléré, } \\
\text { accéléré), } \\
\text { Définition de la relation } \\
\text { liant la vitesse à la distance } \\
\text { d'arrêt. }\end{array}$ & $\begin{array}{c}\text { La translation est exploitée dans le } \\
\text { mouvement de translation (cas du } \\
\text { mouvement de l'ascenseur ou d'une } \\
\text { nacelle par exemple) } \\
\text { Représentation graphique de la variation de } \\
\text { la distance parcourue d en fonction de la } \\
\text { durée } t \text {. } \\
\text { Exploitation de la courbe pour déterminer } \\
\text { la relation liant d et } t \text {. } \\
\text { Exploitation de la relation liant } \mathrm{V} \text {, d et t } \\
\text { pour déterminer la nature du mouvement. } \\
\text { Les relations algébriques modélisant : } \\
\text { la vitesse et la distance de réaction } \mathrm{D}_{\mathrm{r}}=\mathrm{V} \mathrm{x} \\
\mathrm{t}_{\mathrm{r}}\left(\text { (équation du } 1^{\text {er }} \text { degré). }\right. \\
\text { la vitesse et la distance de freinage } \mathrm{D}_{\mathrm{f}}=\mathrm{k} \\
\mathrm{V}^{2}\left(\text { (équation du } 2^{\text {ème degré})}\right. \\
\text { la distance d'arrêt }\left(\mathrm{D}_{\mathrm{a}}\right) \text {, la distance de } \\
\text { réaction }\left(\mathrm{D}_{\mathrm{r}}\right) \text { et la distance de freinage } \\
\left(\mathrm{D}_{\mathrm{f}}\right) \mathrm{D}_{\mathrm{a}}=\mathrm{D}_{\mathrm{r}}+\mathrm{D}_{\mathrm{f}}\end{array}$ \\
\hline
\end{tabular}




\begin{tabular}{|l|c|l|}
\hline & $\begin{array}{c}\text { Dangers de la vitesse et } \\
\text { règles de la sécurité } \\
\text { routière. }\end{array}$ \\
\hline
\end{tabular}

\begin{tabular}{|c|c|c|}
\hline$\overline{A x e}$ & Apprentissages visés & Notions mathématiques mobilisées \\
\hline $\begin{array}{l}\text { Actions } \\
\text { mécaniques }\end{array}$ & $\begin{array}{c}\text { Définition des actions } \\
\text { mécaniques et leur effet } \\
\text { Distinction entre les actions } \\
\text { de contacts et les actions à } \\
\text { distance. }\end{array}$ & \\
\hline $\begin{array}{l}\text { Notion de } \\
\text { force }\end{array}$ & $\begin{array}{l}\text { Définition d'une force. } \\
\text { Mesure de l'intensité d'une } \\
\text { force. } \\
\text { Représentation d'une force } \\
\text { à partir de ses } \\
\text { caractéristiques d'une force. }\end{array}$ & $\begin{array}{l}\text { Modélisation de l'action mécanique par le } \\
\text { vecteur force : } \\
\text { Les coordonnées d'un vecteur } \\
\text { La représentation d'un vecteur à partir de } \\
\text { ses coordonnées. }\end{array}$ \\
\hline $\begin{array}{l}\text { Equilibre } \\
\text { d'un corps } \\
\text { soumis à } \\
\text { l'action de } \\
\text { deux forces }\end{array}$ & $\begin{array}{l}\text { Condition d'équilibre d'un } \\
\text { corps soumis à l'action de } \\
\text { deux forces et son } \\
\text { application }\end{array}$ & $\begin{array}{l}\text { Exploitation de la définition de deux } \\
\text { vecteurs parallèles dans la modélisation de } \\
\text { condition d'équilibre : } \\
\qquad \overrightarrow{\mathrm{F} 1}+\overrightarrow{\mathrm{F} 2}=\overrightarrow{\mathrm{a}}\end{array}$ \\
\hline $\begin{array}{l}\text { Poids et } \\
\text { masse }\end{array}$ & $\begin{array}{l}\text { Caractéristiques du poids } \\
\text { d'un corps solide. } \\
\text { Distinction entre poids et } \\
\text { masse. } \\
\text { La relation entre le poids et } \\
\text { la masse }(\mathrm{P}=\mathrm{m} \times \mathrm{g})\end{array}$ & $\begin{array}{l}\text { La géométrie : des formes géométriques } \\
\text { (triangle, rectangle, carré, cube, cylindre, } \\
\text { etc.) sont exploitées pour déterminer le } \\
\text { centre de gravité. } \\
\text { La proportionnalité pour établir la relation } \\
\text { entre } \mathrm{P} \text { et } \mathrm{m} \text {. }\end{array}$ \\
\hline
\end{tabular}

Tableau 2

\section{Les questionnaires écrits}

Les résultats du questionnaire des mathématiques sont consignés dans le tableau suivant :

\begin{tabular}{|c|c|c|}
\hline $\begin{array}{c}\mathrm{N}^{\circ} \text { de } \\
\text { l'item }\end{array}$ & Items de mathématique & $\begin{array}{c}\% \text { des réponses } \\
\text { correctes }\end{array}$ \\
\hline 1 & $\begin{array}{c}\text { Expression de } \mathrm{b} \text { en fonction de a et c à partir de la } \\
\text { relation } \mathrm{a}=\mathrm{b}+\mathrm{c}\end{array}$ & $71 \%$ \\
\hline 2 & $\begin{array}{c}\text { Expression de } \mathrm{c} \text { en fonction de } \mathrm{a} \text { et } \mathrm{b} \text { à partir de la } \\
\text { relation } \mathrm{a}=\mathrm{b} / \mathrm{c}\end{array}$ & $42 \%$ \\
\hline 3 & Expression de $\mathrm{X}$ à partir de $9 \mathrm{a}=\mathrm{X}^{2}$ & $21 \%$ \\
\hline 4 & Représentation d'un vecteur à partir de ses coordonnées & $56 \%$ \\
\hline 5 & Expression de la somme de deux vecteurs opposés & $51 \%$ \\
\hline
\end{tabular}

\section{Tableau 3}


L'analyse des résultats montre que les sujets semblent avoir plus de facilité à traiter l'équation mobilisant la notion addition/soustraction que celles mobilisant la notion multiplication/division ou la notion racine carrée.

En géométrie vectorielle, plus de la moitié des sujets n'ont pas pu représenter un vecteur à partir de ses coordonnées et exploiter la définition de deux vecteurs parallèles pour déterminer l'équation modélisant la somme de deux vecteurs opposés.

En physique, les résultats sont les suivants :

\begin{tabular}{|c|c|c|}
\hline $\begin{array}{l}\mathrm{N}^{\circ} \text { de } \\
\text { l'item }\end{array}$ & Items de physique & $\begin{array}{l}\% \text { des réponses } \\
\text { correctes }\end{array}$ \\
\hline 1 & $\begin{array}{l}\text { Expression de la distance de réaction }\left(\mathrm{D}_{\mathrm{R}} \text { en fonction de }\right. \\
\text { la distance de freinage }\left(\mathrm{D}_{\mathrm{F}}\right) \text { et de la distance d'arrêt }\left(\mathrm{D}_{\mathrm{A}}\right) \\
\text { à partir de la relation } \mathrm{D}_{\mathrm{A}}=\mathrm{D}_{\mathrm{R}}+\mathrm{D}_{\mathrm{F}}\end{array}$ & $59 \%$ \\
\hline 2 & $\begin{array}{c}\text { Expression du temps t à partir de la relation } \mathrm{V}_{\mathrm{m}}=\mathrm{d} / \mathrm{t} \text {, où } \\
\mathrm{V}_{\mathrm{m}} \text { et } \mathrm{d} \text { désignent respectivement la vitesse moyenne et } \\
\text { la distance parcourue. }\end{array}$ & $59 \%$ \\
\hline 3 & $\begin{array}{c}\text { Expression de la vitesse de freinage en fonction de la } \\
\text { vitesse à partir de la relation } \mathrm{D}_{\mathrm{F}}=2 \mathrm{~V}^{2}\end{array}$ & $16 \%$ \\
\hline 4 & Représentation de la force à partir de ces caractéristiques & $29 \%$ \\
\hline 5 & $\begin{array}{l}\text { Exploitation des conditions d'équilibre d'un corps } \\
\text { soumis à deux forces }\end{array}$ & $33 \%$ \\
\hline
\end{tabular}

Tableau4

Ces résultats indiquent que les sujets ont plus de difficultés à exprimer la vitesse à partir de l'équation $\mathrm{D}_{\mathrm{F}}=2 \mathrm{~V}^{2}$, à représenter la force à partir de ses caractéristiques et à déterminer la relation modélisant la condition d'équilibre d'un corps soumis à deux forces

En comparant les résultats des deux questionnaires, on note que même si chaque item en mathématiques a la même structure et le même type de traitement que son équivalent en physique, certains élèves traitant correctement l'item "i" dans la situation source, rencontrent des difficultés dans la situation cible et inversement:

\begin{tabular}{|c|c|c|}
\hline $\begin{array}{c}\mathrm{N}^{\circ} \text { de } \\
\text { l'item }\end{array}$ & $\begin{array}{c}\text { \% des élèves ayant résolu l'item i en } \\
\text { mathématiques mais pas en physique }\end{array}$ & $\begin{array}{c}\% \text { des élèves ayant résolu l'item i en } \\
\text { physique mais pas en mathématiques }\end{array}$ \\
\hline 1 & $47 \%$ & $36 \%$ \\
\hline 2 & $25 \%$ & $46 \%$ \\
\hline 3 & $64 \%$ & $55 \%$ \\
\hline 4 & $31 \%$ & $38 \%$ \\
\hline 5 & $52 \%$ & $72 \%$ \\
\hline
\end{tabular}

Tableau 5 
$\mathrm{Si}$ on considère les élèves ayant répondu correctement en mathématique, plus de $47 \%$ ayant réussi le traitement des items 1,3 et 5 n'ont pas pu le faire en physique. Pour les items 2 et 4 , en moyenne $28 \%$ des élèves sont concernés par cette problématique.

Ceci semble indiquer, que cette catégorie d'élèves ne réalise pas qu'il s'agit de mobiliser la même notion mathématique dans le traitement de la situation source (mathématiques) et de la situation cible (mécanique) et par conséquent, il leur est difficile de dégager les invariants structurels qui existent entre les deux situations.

Si on considère les sujets ayant répondu correctement en physique, $37 \%$ en moyenne n'ont pas pu traiter les items 1 et 4 en mathématiques et plus de $46 \%$ les items 2,3 et 5 . Le pourcentage est encore plus important pour l'item 5 mobilisant le principe de la somme de deux vecteurs opposés (72\%).

Il semblerait que cette catégorie d'élèves se baserait plutôt sur la mémorisation comme moyen de traitement et non pas sur un raisonnement logique. Cependant, qu'en est-il pour ces sujets qui réussissent à traiter l'item dans les deux situations : traitent-ils ces situations au moyen du raisonnement analogique ou par mémorisation?

\section{Les entretiens individuels avec les élèves}

En écrivant au tableau l'item en physique et son homologue en mathématiques, seulement 3 élèves sur 20 ont remarqué la similitude entre les deux items. Les autres ont été surpris, à la limite du choc, d'apprendre qu'il s'agit de la même notion mathématique mobilisée en physique.

Pour eux, il n'y'a aucun lien entre la démarche de résolution de l'équation $\mathrm{y}=\mathrm{ax}$ et l'expression $\mathrm{d}=\mathrm{Vxt}$ bien que dans les manuels de mathématiques, certaines situations d'apprentissage se basent sur la notion de la vitesse. Nous avons noté les citations des propos des élèves: "non, madame, ça c'est en mathématique alors que là il s'agit de la mécanique", "Ah non professeur, il n'y a pas de "x", pourquoi parler alors de résolution d'équation!", "Regardez Madame, ici c'est écrit caractéristiques d'une force et il s'agit de F ou P ça ne peut pas être la même chose qu'en mathématique : un vecteur a des coordonnées et sont différentes des caractéristiques d'une force, un vecteur c'est $U, V \ldots$

Bien que ces sujets aient de bonnes notes en physique et en mathématiques, les résultats des entrevues révèlent la quasi-absence, dans leur raisonnement, des liens existants entre ces deux disciplines. Il semblerait que les élèves n'ont pas été préparés au transfert de leurs connaissances dans le contexte de la mécanique et aux liens existant entre les mathématiques et la physique et donc à l'articulation de leurs connaissances d'une façon autonome. 


\section{Conclusion}

L'analyse et l'interprétation des résultats des deux questionnaires écrits et des entretiens individuels nous renseignent sur un aspect des difficultés des sujets liées à l'apprentissage des connaissances mathématiques dans la situation source et sur la dynamique de leur mobilisation dans les activités de modélisation des concepts "vitesse" et "force".

Il en ressort que plusieurs facteurs semblent handicaper le transfert des connaissances mathématiques dans des situations mécaniques de modélisation :

- La non-maîtrise, dans la situation source, des connaissances à mobiliser.

- $\quad$ La quasi-absence de reflexes d'articulation et de mise en relation entre la situation source et la situation cible bien que les deux situations présentent une structure logique homologue et qu'elles sont identiques à celles déjà abordées dans les deux disciplines.

- $\quad$ L'adoption de stratégies de mémorisation au dépend de stratégies cognitives propices au transfert.

- Des positions contractuelles reflétant l'existence de barrières quasiimperméables entre les deux disciplines.

Ces résultats obtenus renforcent les conclusions issues de notre précédente étude (Raouf et al) qui relatent la nécessité de considérer la dimension interdisciplinaire dans la formation initiale et continue des enseignants et dans la réforme curriculaire des deux disciplines tout en tenant en compte des spécificités de chaque discipline et du rôle qu'elle joue dans le processus de modélisation .

A partir de ces constats et de nos expériences de terrains, Il semble que les difficultés des élèves identifiées sont des indicateurs d'un dysfonctionnement du système didactique d'une part, et probablement aussi des programmes et des manuels et du système de formation des futurs enseignants. N'est il pas temps de considérer, dans la mise en œuvre de la nouvelle réforme de l'éducation au Maroc le paramètre "transfert" non pas comme un indicateur de performance mais comme un élément moteur d'un apprentissage efficace qu'il soit d'un niveau à un autre ou d'une activité à une autre ou d'un contexte à un autre et aussi en dehors de l'école. Cette approche systémique ne donnerait elle pas, plus de sens aux apprentissages?.

\section{References:}

Bracke, D. (1998).Vers un modèle du transfert : des contraintes à respecter. Revue des sciences de l'éducation, 24(2), 235-266.

Brouillette, N. (2002). Bonification, validation et expérimentation d'un modèle d'intervention en faveur du transfert des apprentissages auprès 
d'élèves à faible rendement scolaire en biologie. Mémoire de maîtrise, Université du Québec à Trois-Rivières.

Chafiqi, F., et Alagui, A. (2011). Réforme éducative au Maroc et refonte des curricula dans les disciplines scientifiques. Carrefours de l'éducation, 3 (HS $\left.\mathrm{n}^{\circ} 1\right), 29-50$

CSEFRS. (2009). Conseil Supérieur de l'Education et de la Formation et de la Recherche Scientifique (Royaume du Maroc). Instance nationale d'évaluation du système d'éducation formation. Rapport thématique sur les résultats du programme national d'évaluation des acquis PNEA 2008

CSEFRS. (2014). Conseil Supérieur de l'Education et de la Formation et de la Recherche Scientifique (Royaume du Maroc). Instance nationale d'évaluation du système d'éducation formation. Rapport analytique sur la mise en æuvre de la charte nationale d'éducation et de formation 2000-2013 Acquis, déficits et défis.

Forcier, P., et Goulet, J.P. (1996). Un problème et un mystère : Le transfert des apprentissages. Pédagogie collégiale. 10 (2), 30-32.

Gagné, R.M. (1962). The acquisition of knowledge. Psychological Review, 69, 355-365.

Gick, M. L., et Holyoak, K. J. (1980). Analogical problem solving. Cognitive Psychology, 12, 306-355.

Gick, M. L., et Holyoak, K. J. (1983). Schema Induction and Analogical Transfer. Cognitive Psychology, 15, 1-38.

Gick, M.L., et Holyoak, K.J. (1987). The cognitive basis of knowledge transfer. In Cormier, S.M., et Hagman, J.D. (dir.), Transfer on Learning. Contemporary Research and Applications, 9-46. San Diego (CA): Academic Press.

Haskell, R.E. (2001). Transfer of learning : Cognition, instruction, and reasoning. San Diego, CA : Academic Press.

Lauzon, F. ( 2000). Comment aider les élèves à intégrer et à transférer leurs apprentissages ?: Proposition d'un itinéraire en cinq actions-réflexions. Pédagogie collégiale 14 (2), 34-40.

Mc.Keough, A., Lupart, J., et Marini, A. (dir.). (1995). Teaching for transfer : Fostering generalization in learning. Mahwah, NJ : Lawrence Erlbaum

Meirieu, P. (1991). le choix d'éduquer. Ethique et pédagogie, Paris, ESF éditeur.

Mendelson, P. (1990). La notion de transfert d'apprentissage en psychologie cognitive. Cahier pédagogique, 281. 23-25.

MENFP. Ministère de l'Education Nationale et de la Formation Professionnelle. Rapports des résultats des consultations nationales sur l'école marocaine (2014). Version arabe disponible en ligne : http://www.men.gov.ma/frum-rapport-concertaions/Pages/bilanconcertations.aspx 
Moffet, J.D. (1995). Des stratégies pour favoriser le transfert des connaissances en écriture au collégial. Revue des sciences de l'éducation, XXI(1), 95-120.

Péladeau, N., Forget, J ., et Gagné F. (2005) ; Le transfert des apprentissages et la réforme de l'éducation au Québec: quelques mises au point. Revue des sciences de l'éducation, 31(1), 187-209.

Perrenoud, Ph. (1997). Vers des pratiques pédagogiques favorisant le transfert des acquis scolaires hors de l'école. Pédagogie collégiale (Québec), 10( 3), 5-16.

Perrenoud, Ph. (2000). D'une métaphore à l'autre: transférer ou mobiliser ses connaissances. In Dolz, J et Ollagnier E. (dir). L'énigme de la compétence en éducation, Bruxelles, De Boek, coll. Raisons éducatives, 45-60.

Presseau, A., et Frenay, M. (2004). Le transfert des apprentissages, comprendre pour mieux intervenir. Les presses de l'université Laval, SAINTE-FOY, Québec, Canada

Raouf, K., Radi, M., Bellazaar, I., Moussetad, M., et Talbi, M. soumis pour publication.

Singley, M.K., et Anderson, J.R. (1989). The transfer of cognitive skill. Cambridge, MA : Harvard University Press.

Tardif, J. (1992). Pour un enseignement stratégique. L'apport de la psychologie cognitive. Montréal : Éditions Logiques.

Tardif, J., et Meirieu, P. (1996). Stratégie pour favoriser le transfert des connaissances. Vie pédagogique, 98, 4-7

Tardif, J et Presseau, A, (1998). Quelques contributions de la recherche pour favoriser le transfert des apprentissages. Vie pédagogique, 108, 39-44.

Tardif, J. (1999). Le transfert des apprentissages. Montréal : Éditions Logiques.

THORNDIKE, E.K. (1906). Principles of Teaching. New York: A. G. Seiler. In Argot, L. (2000). Organizational Behavior and Human Decision Processes. 82(1), 150-169.

TIMSS. (2011); Trends in International Mathematics and Science Survey.

Toussaint, J. (1996). Didactique appliquée de la physique-chimie. Nathan Pédagogie.

UNESCO. (2014). Rapport mondial de suivi sur l'EPT 2013-2014 : Enseigner et apprendre: Atteindre la qualité pour tous.

\section{Remerciements:}

Sincères remerciements à Pr Squalli Hassane (Département de Pédagogie, Faculté d'Éducation, Université de Sherbrooke) pour ses remarques pertinentes que nous avons prises en compte dans la rédaction de cet article. 